\title{
Efeito da Formação de Classes de Estímulos Equivalentes e de Procedimentos de Facilitação na Emergência de Analogia*
}

\author{
Madeleine Reinert Marcelino** (D), Ana Arantes (D), \& Nassim Chamel Elias (D) \\ Universidade Federal de São Carlos, São Carlos, SP, Brasil
}

\begin{abstract}
RESUMO - Buscou-se verificar os efeitos da formação de classes de estímulos equivalentes e de dois procedimentos de facilitação na emergência de respostas de analogia, comparando os resultados obtidos nos testes de analogia de acordo com o procedimento de facilitação e com o fornecimento de instrução. Participaram 30 universitários. Foram ensinadas as relações $\mathrm{AB}$ e $\mathrm{AC}$ entre figuras abstratas e testadas as relações de simetria, transitividade e analogia. Os participantes que não apresentaram essas relações foram expostos aos procedimentos de facilitação. Doze participantes responderam por analogia antes do procedimento de facilitação, cinco de oito (62\%), após Facilitação 1 e três de dez (33\%), após Facilitação 2. Dos sete participantes expostos à instrução, seis responderam por analogia. A formação das classes não foi suficiente para a emergência das respostas de analogia e alguns participantes podem ter respondido ao Teste de Analogia sob controle de apenas alguns aspectos dos pares de estímulos.
\end{abstract}

PALAVRAS-CHAVES: equivalência de estímulos, raciocínio analógico, procedimento de facilitação, instruções

\section{Effect of Equivalent Stimulus Class Formation and Facilitation Procedures on the Emergence of Analogy}

\begin{abstract}
The goals were to verify the effects of the formation of equivalent stimulus classes and two facilitation procedures on the emergence of analogy responses and to compare the results obtained in the analogy tests according to the facilitation procedure and the provision of instruction. Participants were 30 undergraduate students. AB and AC relations were taught with abstract pictures, then symmetry, transitivity, and analogy relations were tested. Participants who did not show these relations were exposed to the facilitation procedures. Twelve participants responded by analogy before the facilitation procedure, five of eight (62\%) after Facilitation 1 and three of ten (33\%) after Facilitation 2 . Of the seven participants exposed to the instruction, six responded by analogy. Class formation was not enough for the emergence of analogy responses, and some participants might have responded to the Analogy Test under the control of only some aspects of the stimulus pairs.
\end{abstract}

KEYWORDS: stimulus equivalence, analogical reasoning, facilitation procedure, instructions

Analogia é um conceito amplo que vem do Grego ana logon, que pode significar de acordo com uma proporção. A analogia tem um papel significativo na resolução de problemas, tomada de decisão, criatividade, comunicação, entre outros (Kolodner, 1997). Em função dessas características, ela está presente na linguagem diária, no senso comum, na ciência, na filosofia e nas humanidades. Originalmente, os matemáticos gregos usaram a palavra "analogia" para denotar similaridade em relações proporcionais ou de função. Segundo Lipkens e
Hayes (2009), Aristóteles declarou o esquema clássico de proporção A:B:C:D e as fórmulas dos dois tipos de analogia: "Assim como A está em/para B, C está em/para D".

Skinner (1957) definiu analogia como um processo de abstração, por meio da extensão metafórica do tato. Pela definição de Skinner, o tato é definido como uma resposta verbal sob controle de estímulos antecedentes não-verbais, como objetos, eventos ou suas propriedades, e de reforçadores generalizados. Exemplos de tato são dizer "gato" ao ver um gato ou dizer "o dia está ensolarado" ao

\footnotetext{
* Apoio: FAPESP; CAPES

** E-mail: madeleine.marcelino@gmail.com

- Submetido: 28/12/2016; Revisado: 11/03/2018; Aceito: 21/05/2018.
} 
olhar para o céu. Skinner propõe que há vários tipos de tato estendido ao discutir a emergência de respostas não reforçadas diretamente, como quando um novo estímulo não-verbal evoca uma resposta de tato previamente reforçada na presença de outro estímulo não-verbal, por apresentarem características físicas ou funcionais semelhantes. Tatos estendidos do tipo analogia são respostas verbais de tato em que uma topografia previamente estabelecida na presença de uma relação entre estímulos é reforçada na presença de outros estímulos que se relacionam de maneira semelhante. No caso da resposta de analogia, as características determinantes para extensão do controle são as relações entre estímulos, de modo que um par ou conjunto de estímulos relacionados entre si controlam a mesma resposta que outro par ou conjunto de estímulos que apresentam o mesmo tipo de relação física ou funcional.

Autores da RFT (do inglês Relational Frame Theory Teoria dos Quadros Relacionais; Stewart \& Barnes-Holmes, 2001; Stewart, Barnes-Holmes, \& Roche, 2004) diferenciam os fenômenos da analogia e da metáfora pela direcionalidade das relações derivadas. Analogias são bidirecionais, enquanto metáforas são unidirecionais (Stewart \& BarnesHolmes, 2001). Considere os seguintes exemplos: "Gatos são ditadores" e "O átomo é como o sistema solar". O primeiro é um exemplo de metáfora, pois características do segundo termo (demandas, exigências) são transferidas para o primeiro; entretanto, se os termos forem invertidos, "ditadores são gatos", esse efeito deixa de existir, pois as propriedades comuns do segundo termo (gato), como carinhosos e fofos, não são obviamente transferidas para o primeiro (ditador). No outro exemplo, tanto o átomo como o sistema solar possuem um núcleo central e elétrons/planetas orbitais, então, se os termos forem invertidos, "O sistema solar é como o átomo", a analogia continua válida ${ }^{1}$.

Segundo Stewart e Barnes-Holmes (2001), a RFT é uma abordagem sobre linguagem e cognição humanas que trata esses fenômenos como exemplos de responder relacional arbitrariamente aplicável. De acordo com a RFT, os seres humanos verbais, após uma exposição prolongada a certas contingências de reforçamento que operam dentro da comunidade verbal, também demonstram responder com base nas relações derivadas ou arbitrariamente aplicáveis. Estas relações não são definidas pelas propriedades físicas dos estímulos relacionados, mas por dicas contextuais adicionais.

Segundo a perspectiva da RFT, uma das propriedades importantes de um símbolo é que as suas funções psicológicas são baseadas, em certa medida, na transferência de funções, por meio, por exemplo, das relações de equivalência ou de quadros de coordenação (Stewart \& Barnes-Holmes, 2001).

1 Nos exemplos apresentados, a história de reforçamento que estabeleceu tais repertórios pode ter se dado por outras vias (e.g., por encadeamento intraverbal de respostas), caso em que não haveria necessidade de que o falante emitisse respostas generalizadas diante de relações de analogia. O exemplo se aplica nos casos em que o repertório foi estabelecido pelo processo de extensão de tato descrito por Skinner (1957).
Suponha, por exemplo, que uma menina experimenta um copo de suco de laranja (Estímulo A) pela primeira vez e aprecie o seu sabor. Em seguida, uma amiga lhe diz que o suco de laranja é como o suco de tangerina (Estímulo B). Se alguém posteriormente perguntar para a menina "Você quer suco de tangerina?", ela pode responder positivamente. Essa transferência da função apetitiva baseia-se diretamente na função psicológica estabelecida pela palavra laranja, que foi pareada com um suco de laranja real, e a relação simétrica derivada entre as palavras laranja e tangerina (isto é, tangerina é como laranja). A RFT tem utilizado os conceitos de equivalência e transferência de função entre estímulos para analisar a metáfora e a analogia.

Barnes, Hegarty e Smeets (1997) originalmente definiram analogia como relacionar relações de equivalência a outras relações de equivalência (A1B1 - C1D1, sendo que as letras representam estímulos distintos e o número representa a classe) e relações de não-equivalência a outras relações de não-equivalência (A1B2 - C1D2). Segundo Stewart, Barnes-Holmes, Hayes e Lipkens (2001), relacionar relações é particularmente importante porque pode explicar, em parte, $\mathrm{o}$ grau de geratividade da linguagem e como a linguagem pode levar à criação de novos conceitos, precisos mas abstratos.

Sidman (1971) publicou um artigo sobre repertórios emergentes ou derivados, no qual ensinou um jovem com microcefalia, por meio de discriminações condicionais, a selecionar palavras impressas na presença das palavras ditadas correspondentes (leitura receptiva). Esse jovem já apresentava, em seu repertório, relações entre palavras ditadas e figuras. Em função do ensino ele aprendeu a relacionar as palavras impressas com as figuras correspondentes e a ler oralmente essas palavras impressas (leitura expressiva) sem que essas relações fossem diretamente ensinadas. Barnes et al. (1997) demonstraram que esse tipo de treino ainda poderia produzir outros resultados. Alguns participantes emparelhavam, consistentemente, estímulos modelos compostos por dois estímulos equivalentes ou não-equivalentes com estímulos comparações compostos por dois estímulos equivalentes ou não-equivalentes, respectivamente. Em geral, nesses estudos, são ensinadas relações arbitrárias entre estímulos (por exemplo, $\mathrm{AB}$ e $\mathrm{BC}$ ) e depois verifica-se que os participantes derivam relações entre estímulos não ensinadas diretamente (BA, CB, AC e CA) e relações entre relações, como, por exemplo, diante da relação A1B1 (estímulos equivalentes) o participante seleciona $\mathrm{B} 2 \mathrm{C} 2$ (também equivalentes), em detrimento de um par não equivalente (por exemplo, B2C3). Nesse último exemplo, pode-se interpretar que A1 está para $\mathrm{B} 1$ assim como B2 está para $\mathrm{C} 2$. Esses autores têm chamado essa relação de Equivalência-Equivalência (Eq-Eq). Por outro lado, se diante da relação A1B2 (estímulos não-equivalentes) o participante seleciona $\mathrm{B} 2 \mathrm{C} 3$ (também não-equivalentes), em detrimento de um par equivalente (por exemplo, B2C2), essa nova relação é do tipo NãoEquivalência-NãoEquivalência (NEq-NEq). O modelo experimental de Eq-Eq e NEq-NEq é interpretado como um modelo analítico-comportamental 
para estudo de extensões de tato do tipo metáfora e analogia (e.g., Carpentier, Smeets, Barnes-Holmes, \& Stewart, 2004; Stewart et al., 2004; Stewart, Barnes-Holmes, Roche, \& Smeets, 2001).

Pérez, García e Gomez (2011) tinham como objetivo verificar a emergência de relações Eq-Eq e NEq-NEq após o estabelecimento de três classes de estímulos equivalentes, com três membros em cada classe. No primeiro experimento, 12 adultos foram expostos à treinos com tarefas de MTS (do inglês Matching to Sample, Escolha de Acordo com o Modelo). $\mathrm{O}$ treino consistia de blocos em que as relações $\mathrm{AB}$ eram reforçadas (A1B1, A2B2, A3B3); em seguida, as relações $\mathrm{AC}(\mathrm{A} 1 \mathrm{C} 1, \mathrm{~A} 2 \mathrm{C} 2, \mathrm{~A} 3 \mathrm{C} 3)$ eram reforçadas em um bloco separado; e, finalmente, um bloco em que relações $\mathrm{AB}$ e AC eram apresentadas de forma intercalada. Após alcance de critério nos três blocos, um teste parcial de equivalência das relações emergentes $\mathrm{CB}$ (C1B1, C2B2, C3B3), com 15 tentativas, foi introduzido. Os participantes que acertaram pelo menos 13 tentativas das relações $\mathrm{CB}$ foram expostos aos testes de Eq-Eq e NEq-NEq para verificar se apresentavam essas relações sem ensino direto. Os oito dos 12 participantes, que não demonstraram a emergência desse novo repertório, foram expostos novamente aos testes Eq-Eq e NEq-NEq após um intervalo de $4 \mathrm{~min}$. Os resultados mostraram que a repetição do teste não foi suficiente para melhorar o desempenho de nenhum desses oito participantes. No segundo experimento, 42 adultos passaram por um procedimento semelhante, porém, entre os dois blocos de teste das relações Eq-Eq e NEq-NEq, foi introduzido um procedimento de facilitação. Esse procedimento consistia em tarefas de MTS nas quais os estímulos eram pares formados por estímulos dos conjuntos $\mathrm{A}, \mathrm{B} \mathrm{e} \mathrm{C}$ e pares de formas geométricas iguais ou diferentes (dois círculos iguais ou um quadrado e um triângulo). Se o estímulo modelo fosse um par de figuras geométricas iguais, escolher um par de estímulos equivalentes (por exemplo, A1B1) produzia o reforço; se o estímulo modelo fosse um par de figuras geométricas diferentes, escolher um par de estímulos não-equivalentes (por exemplo, A1B2) era reforçado. Os resultados demonstraram que apenas $40 \%$ dos participantes passaram no segundo teste das relações Eq-Eq e NEq-NEq. Entretanto, o procedimento de facilitação foi mais eficiente que a repetição simples do teste, como realizado no Experimento 1, em que nenhum participante passou no segundo teste.
Nos dois experimentos de Pérez et al. (2011), o desempenho nos testes das relações de Eq-Eq e NEq-NEq foi apresentado pela primeira vez após o estabelecimento das classes, ou seja, não há dados de linha de base para serem comparados com os dados do teste. Além disso, os autores testaram somente as relações derivadas $\mathrm{CB}, \mathrm{o}$ que não garante, segundo o paradigma de equivalência de estímulos (Sidman \& Tailby, 1982), que os estímulos pertencem a uma mesma classe; seriam necessários, pelo menos, os testes de simetria (relações BA e CA) e o outro teste de transitividade (relações BC). Finalmente, como o procedimento de facilitação com figuras geométricas gerou respostas de analogia para apenas $40 \%$ dos participantes, os efeitos de outros procedimentos de facilitação poderiam ser experimentalmente testados. Procedimentos de facilitação são introduzidos quando o procedimento programado não é suficiente para que os participantes apresentem respostas emergentes durante os testes. Stokes e Baer (1977) propuseram a estratégia de garantir a generalização (ou emergência de respostas diante de novos estímulos) com o uso de procedimentos de modificação sequencial e sistemática das condições em que a resposta deveria ser emitida generalizadamente.

Portanto, o objetivo do presente estudo foi verificar os efeitos da formação de classes de estímulos equivalentes e da introdução de dois tipos de procedimentos de facilitação na emergência de respostas de analogia para relações EqEq e NEq-NEq não ensinadas diretamente. Os objetivos secundários foram comparar os resultados obtidos pelos participantes nos testes de analogia de acordo com o procedimento de facilitação a que foram expostos e com o fornecimento de instrução. Foram introduzidas as seguintes mudanças em relação ao procedimento de Pérez et al. (2001): (a) múltiplas sondagens das respostas de analogia, intercaladas entre relações de ensino e relações emergentes, para verificar se alguma condição experimental específica seria suficiente para a emergência dessas respostas; (b) testes das relações emergentes de simetria e transitividade; (c) dois procedimentos de facilitação, um idêntico ao utilizado por Pérez et al. e outro em que os estímulos de comparação eram estímulos simples, como nas tentativas de ensino; e (d) a introdução de uma instrução verbal caso os procedimentos de facilitação não levassem o participante a responder corretamente pelo menos $80 \%$ do teste de analogia.

\section{MÉTODO}

\section{Participantes}

Foram participantes desse estudo 30 adultos universitários (P1 a P29), com idades entre 17 e 40 anos, de ambos os gêneros, que não tinham conhecimento prévio dos propósitos do estudo.

\section{Ambiente e Equipamentos}

As sessões ocorreram em uma sala equipada com uma mesa, duas cadeiras e um computador PC com sistema operacional Windows, mouse e teclado. Cada sessão teve a duração de aproximadamente $60 \mathrm{~min}$ ou até que o participante 
demonstrasse sinais de cansaço ou solicitasse a interrupção do trabalho. O programa computacional MestreLibras (Elias \& Goyos, 2010) foi utilizado para programar e realizar a apresentação dos estímulos, apresentar as consequências e armazenar os dados das tarefas (descritas a seguir). Durante as sessões de ensino e de teste, o participante sentou-se em uma cadeira, em frente ao computador. A experimentadora esteve presente na mesma sala somente para iniciar cada condição experimental e fornecer as instruções mínimas necessárias.

\section{Estímulos Experimentais}

Os estímulos foram nove figuras abstratas subdivididas em três conjuntos (Conjuntos A, B e C) retiradas do estudo de Pérez et al. (2011), duas figuras geométricas (Conjunto D) e figuras com os sinais de igual e de diferente (Conjunto X) (ver Tabela 1).

\section{Procedimento}

Delineamento experimental. Foi utilizado um delineamento com múltiplas sondagens das relações de analogia, conforme pode ser visto na Tabela 2 .

Tarefas de escolha de acordo com o modelo (MTS). Cada tentativa foi iniciada com a apresentação de um estímulo modelo simples ou composto, de acordo com a condição experimental (detalhadas a seguir), centralizado na metade superior do monitor do computador. Assim que o participante clicava com o botão esquerdo do mouse sobre o estímulo modelo, o programa apresentava dois ou três estímulos de comparação, de acordo com a condição experimental, na metade inferior da tela, um ao lado do outro, equidistantes entre si. O participante deveria, então, clicar com o mouse no estímulo comparação que considerasse correto. Nos blocos de ensino, a escolha de um estímulo de comparação produzia a apresentação de consequências diferenciais para respostas corretas e incorretas, indicando o término da tentativa. As respostas corretas produziam a frase "Você acertou" na tela do computador; as respostas incorretas produziam uma tela preta. Nos blocos de teste, não havia consequências diferenciais para respostas corretas ou incorretas. Nos dois tipos de blocos, havia um intervalo entre as tentativas de 2 s. Antes do início de cada bloco, a pesquisadora avisava o participante se haveria ou não consequências para suas respostas. Cada estímulo modelo foi apresentado o mesmo número de vezes dentro de cada um dos blocos, tanto de ensino como de teste, distribuídos randomicamente, para cada posição do estímulo comparação correto. Os estímulos de comparação corretos não foram apresentados na mesma posição por mais de duas tentativas consecutivas, e nenhum estímulo modelo foi repetido por mais de duas tentativas consecutivamente.

Teste de analogia. Nessa condição, foram apresentadas tarefas de MTS com 18 tentativas, sendo nove Eq-Eq e

Tabela 1

Estímulos experimentais divididos em três conjuntos e três classes

\begin{tabular}{|c|c|c|c|}
\hline \multirow{2}{*}{ Conjuntos } & \multicolumn{3}{|c|}{ Classes } \\
\hline & 1 & 2 & 3 \\
\hline \multicolumn{4}{|l|}{$\mathbf{A}$} \\
\hline \multicolumn{4}{|l|}{ B } \\
\hline \multicolumn{4}{|l|}{ C } \\
\hline D & & & \\
\hline $\mathbf{X}$ & & & \\
\hline
\end{tabular}


Tabela 2

Condições experimentais e as características de cada condição

\begin{tabular}{|c|c|c|c|}
\hline Condição & Estímulos & Número de tentativas & Critério* \\
\hline Teste de Analogia & Pares dos conjuntos A, B e C & 18 (9 Eq-Eq e 9 NEq-NEq) & $<=60 \%$ \\
\hline Ensino das Relações AB & $\mathrm{A} 1 \mathrm{~B} 1, \mathrm{~A} 2 \mathrm{~B} 2, \mathrm{~A} 3 \mathrm{~B} 3$ & 9 & $>=80 \%$ \\
\hline Ensino das Relações AC & $\mathrm{A} 1 \mathrm{C} 1, \mathrm{~A} 2 \mathrm{C} 2, \mathrm{~A} 3 \mathrm{C} 3$ & 9 & $>=80 \%$ \\
\hline $\begin{array}{l}\text { Ensino das Relações } \\
\mathrm{AB} / \mathrm{AC} \text { intercaladas }\end{array}$ & $\begin{array}{c}\mathrm{A} 1 \mathrm{~B} 1, \mathrm{~A} 2 \mathrm{~B} 2, \mathrm{~A} 3 \mathrm{~B} 3, \mathrm{~A} 1 \mathrm{C} 1, \mathrm{~A} 2 \mathrm{C} 2 \\
\mathrm{~A} 3 \mathrm{C} 3\end{array}$ & 9 & $>=80 \%$ \\
\hline Teste de Analogia & Pares dos conjuntos $\mathrm{A}, \mathrm{B}$ e C & 18 (9 Eq-Eq e 9 NEq-NEq) & $<80 \%$ \\
\hline Teste das Relações BA & $\mathrm{B} 1 \mathrm{~A} 1, \mathrm{~B} 2 \mathrm{~A} 2, \mathrm{~B} 3 \mathrm{~A} 3$ & 9 & $>=90 \%$ \\
\hline Teste das Relações CA & $\mathrm{C} 1 \mathrm{~A} 1, \mathrm{C} 2 \mathrm{~A} 2, \mathrm{C} 3 \mathrm{~A} 3$ & 9 & $>=90 \%$ \\
\hline Teste das Relações BC & $\mathrm{B} 1 \mathrm{C} 1, \mathrm{~B} 2 \mathrm{C} 2, \mathrm{~B} 3 \mathrm{C} 3$ & 9 & $>=90 \%$ \\
\hline Teste das Relações CB & $\mathrm{C} 1 \mathrm{~B} 1, \mathrm{C} 2 \mathrm{~B} 2, \mathrm{C} 3 \mathrm{~B} 3$ & 9 & $>=90 \%$ \\
\hline Teste de Analogia & Pares dos conjuntos A, B e C & 18 (9 Eq-Eq e 9 NEq-NEq) & $<80 \%$ \\
\hline $\begin{array}{l}\text { Ensino com Procedimento } \\
\text { de Facilitação }\end{array}$ & $\begin{array}{c}\text { Pares dos conjuntos A, B, C e D e } \\
\text { estímulos do conjunto X }\end{array}$ & 18 & $<80 \%$ \\
\hline Teste de Analogia & Pares dos conjuntos A, B e C & 18 (9 Eq-Eq e 9 NEq-NEq) & $<80 \%$ \\
\hline Instrução + Teste de Analogia & Pares dos conjuntos $\mathrm{A}, \mathrm{B}$ e $\mathrm{C}$ & 18 (9 Eq-Eq e 9 NEq-NEq) & - \\
\hline
\end{tabular}

* O critério refere-se à quantidade de respostas corretas para prosseguir para a próxima condição experimental.

nove NEq-NEq (ver Tabela 3). Os estímulos modelos e de comparação foram figuras compostas por duas figuras retiradas da Tabela 1 (por exemplo, A1B1, B2C3). As respostas corretas nessa condição foram definidas como a escolha do estímulo de comparação composto por duas figuras da mesma classe quando o estímulo modelo fosse composto por duas figuras da mesma classe (Eq-Eq) ou a escolha do estímulo de comparação composto por duas figuras de classes diferentes quando o estímulo modelo fosse composto por duas figuras de classes diferentes (NEq-NEq). A Figura 1 apresenta um exemplo desses tipos de tentativas.

Tabela 3

Bloco de tentativas do teste de analogia.

\begin{tabular}{|c|c|c|c|c|}
\hline \multirow{3}{*}{$\begin{array}{l}\text { Tentativa } \\
1 .\end{array}$} & \multicolumn{3}{|c|}{ Estímulo } & \multirow{3}{*}{$\begin{array}{c}\text { Tipo } \\
\text { NEq-NEq }\end{array}$} \\
\hline & \multirow{2}{*}{$\begin{array}{c}\text { Modelo } \\
\text { A3B2 }\end{array}$} & \multicolumn{2}{|c|}{ Comparações* } & \\
\hline & & $\mathrm{B} 1 \mathrm{C} 1$ & C2B3 & \\
\hline 2. & $\mathrm{C} 3 \mathrm{~A} 3$ & B2C2 & $\mathrm{A} 2 \mathrm{~B} 1$ & $\mathrm{Eq}-\mathrm{Eq}$ \\
\hline 3. & $\mathrm{~B} 3 \mathrm{C} 3$ & $\mathrm{C} 2 \mathrm{~B} 2$ & $\mathrm{~A} 3 \mathrm{C} 1$ & $\mathrm{Eq}-\mathrm{Eq}$ \\
\hline 4. & A1B3 & $\mathrm{B} 1 \mathrm{C} 1$ & C3B2 & NEq-NEq \\
\hline 5. & $\mathrm{~A} 1 \mathrm{C} 1$ & B2C2 & A2B3 & Eq-Eq \\
\hline 6. & $\mathrm{C} 2 \mathrm{~A} 2$ & C3B1 & C1A1 & $\mathrm{Eq}-\mathrm{Eq}$ \\
\hline 7. & $\mathrm{~B} 1 \mathrm{C} 3$ & $\mathrm{~A} 1 \mathrm{C} 1$ & B3A2 & NEq-NEq \\
\hline 8. & A3B1 & B3C1 & $\mathrm{B} 2 \mathrm{C} 2$ & NEq-NEq \\
\hline 9. & $\mathrm{~B} 2 \mathrm{~A} 2$ & $\mathrm{~B} 1 \mathrm{C} 2$ & B3A3 & Eq-Eq \\
\hline 10. & $\mathrm{~A} 2 \mathrm{C} 2$ & $\mathrm{~B} 2 \mathrm{C} 3$ & A3B3 & $\mathrm{Eq}-\mathrm{Eq}$ \\
\hline 11. & $\mathrm{C} 3 \mathrm{~A} 2$ & C2A1 & C1B1 & NEq-NEq \\
\hline 12. & $\mathrm{~B} 2 \mathrm{~A} 3$ & $\mathrm{~A} 2 \mathrm{C} 2$ & B3A1 & NEq-NEq \\
\hline 13. & B1A1 & C3B3 & $\mathrm{C} 1 \mathrm{~B} 2$ & Eq-Eq \\
\hline 14. & C1B1 & A2B2 & $\mathrm{A} 1 \mathrm{C} 3$ & $\mathrm{Eq}-\mathrm{Eq}$ \\
\hline 15. & B1A3 & B3C 3 & B2A1 & NEq-NEq \\
\hline 16. & $\mathrm{~A} 2 \mathrm{C} 1$ & $\mathrm{C} 2 \mathrm{~A} 3$ & $\mathrm{~A} 1 \mathrm{~B} 1$ & NEq-NEq \\
\hline 17. & C3A1 & $\mathrm{A} 2 \mathrm{~B} 2$ & C1A3 & NEq-NEq \\
\hline 18. & $\mathrm{~A} 3 \mathrm{C} 3$ & B1C1 & $\mathrm{A} 1 \mathrm{C} 2$ & Eq-Eq \\
\hline
\end{tabular}

* Os estímulos de comparação corretos estão em negrito. 

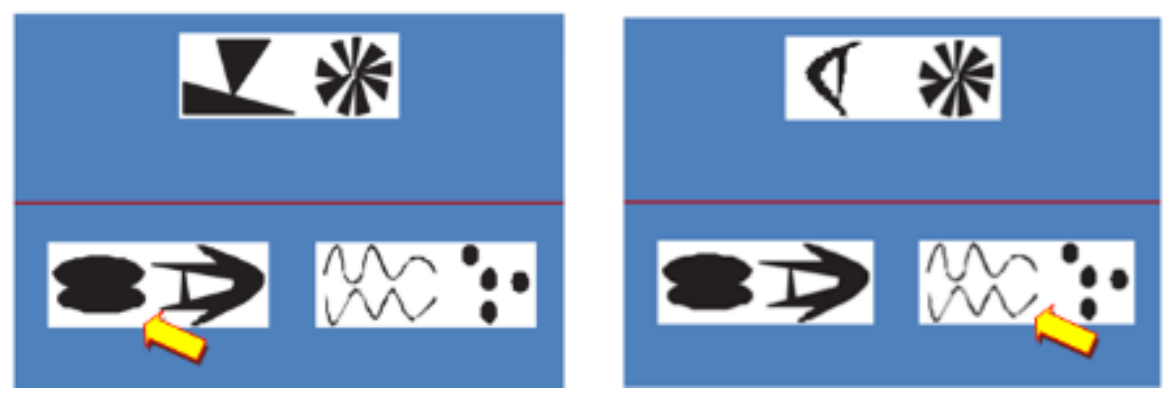

Figura 1. Exemplos de tentativa de teste de analogia. À esquerda, um exemplo de Eq-Eq (A1B1 - A3B3). À direita, um exemplo de NEq-NEq (A2B1 - C1B2). As setas amarelas indicam o estímulo de comparação correto.

Ensino das relações entre estímulos simples. Os participantes que obtiveram desempenho igual ou inferior a $60 \%$ de acertos no primeiro Teste de Analogia prosseguiram no experimento e foram expostos a essa condição; para os outros, o experimento era encerrado. Primeiramente foi introduzido o ensino, por tentativa e erro, das relações A1B1, A2B2, A3B3 com blocos de nove tentativas de MTS (três tentativas para cada relação), sendo que, em cada tentativa, um estímulo do conjunto A foi apresentado como modelo e os três estímulos do conjunto B como comparações. Quando o participante alcançava o critério de $80 \%$ de acertos em um bloco, era iniciado o ensino das relações $\mathrm{A} 1 \mathrm{C} 1, \mathrm{~A} 2 \mathrm{C} 2, \mathrm{~A} 3 \mathrm{C} 3$, realizado da mesma maneira que o ensino das relações $\mathrm{AB}$. Após o alcance de critério nas relações $\mathrm{AC}$, foi introduzido o ensino das relações $\mathrm{AB}$ e $\mathrm{AC}$ apresentadas randomicamente e intercaladas num mesmo bloco com seis tentativas $A B$ e seis tentativas $\mathrm{AC}$ até alcance de critério de $80 \%$ de acertos. Para cada tipo de treino de relações condicionais (AB, AC), foram utilizados dois blocos distintos, em que os estímulos modelos eram apresentados em sequências distintas e os de comparação corretos eram apresentados em posições distintas. Caso o participante não atingisse o critério em até quatro blocos para cada treino $(\mathrm{AB}, \mathrm{AC}$ ou $\mathrm{AB} / \mathrm{AC}$ intercaladas), era dispensado do estudo.

Teste das relações de equivalência. Os participantes que demonstraram desempenho inferior a $80 \%$ de acertos no segundo Teste de Analogia prosseguiram no experimento e foram expostos a essa condição, em que eram testadas as relações não ensinadas diretamente $\mathrm{BA}(\mathrm{B} 1 \mathrm{~A} 1, \mathrm{~B} 2 \mathrm{~A} 2$, $\mathrm{B} 3 \mathrm{~A} 3), \mathrm{CA}(\mathrm{C} 1 \mathrm{~A} 1, \mathrm{C} 2 \mathrm{~A} 2, \mathrm{C} 3 \mathrm{~A} 3), \mathrm{BC}(\mathrm{B} 1 \mathrm{C} 1, \mathrm{~B} 2 \mathrm{C} 2$, $\mathrm{B} 3 \mathrm{C} 3)$ e $\mathrm{CB}$ (C1B1, C2B2, C3B3); para os outros, o experimento era encerrado. Para cada relação, foi utilizado um bloco com nove tentativas, três de cada classe definida experimentalmente, sem a apresentação de consequências diferenciais para respostas corretas e incorretas. Se o participante não obtivesse $90 \%$ de acertos em pelo menos três desses testes, o ensino das relações $\mathrm{AB}$ e $\mathrm{AC}$ e teste das relações de equivalência eram reapresentados até que $o$ critério fosse atingido.

Procedimento de facilitação 1 com figuras geométricas. Metade dos participantes (Grupo 1), que não demonstrou pelo menos $80 \%$ de acertos no Teste de Analogia aplicado após o teste das relações de equivalência, foi exposta a esse procedimento com tarefas de MTS. Cada bloco foi composto por 18 tentativas, sendo três tentativas das relações $\mathrm{AB}$ (A1B1, A2B2, A3B3) e três das relações AC (A1C1, A2C2, $\mathrm{A} 3 \mathrm{C} 3$ ) para verificar manutenção das relações ensinadas. As 12 tentativas restantes foram compostas por seis tentativas do tipo Eq-Eq e seis tentativas do tipo NEq-NEq, em que os estímulos modelos foram figuras compostas por duas figuras dos conjuntos $\mathrm{A}, \mathrm{B}$ e C e os estímulos de comparação foram compostos por duas figuras geométricas do conjunto D. A Figura 2 (duas figuras superiores) apresenta exemplos desses tipos de tentativas. O critério de desempenho foi de $80 \%$ de acertos em um bloco. Caso o participante não atingisse o critério em até quatro blocos, era dispensado do estudo. Foram utilizadas seis figuras compostas (três com estímulos equivalentes e três com estímulos não equivalentes) já utilizadas no Teste de Analogia e seis figuras compostas novas.

Procedimento de facilitação $2 \mathrm{com}$ sinais. A outra metade dos participantes (Grupo 2), que não demonstrou pelo menos $80 \%$ de acertos no Teste de Analogia aplicado após o teste das relações de equivalência, foi exposta a esse procedimento com tarefas de MTS. Os blocos foram semelhantes ao procedimento de Facilitação 1, exceto que os estímulos de comparação foram os sinais do conjunto X. A Figura 2 (duas figuras inferiores) apresenta exemplos desses tipos de tentativas. O critério de desempenho foi de $80 \%$ de acertos em um bloco. Caso o participante não atingisse o critério em até quatro blocos, era dispensado do estudo.

Teste de analogia com instrução verbal. Se mesmo após o procedimento de facilitação o participante não atingisse o critério no Teste de Analogia, era dada uma instrução verbal. A experimentadora, então, iniciava a tarefa do Teste de Analogia e, na primeira tentativa, perguntava ao participante "Essas duas figuras na parte de cima da tela são equivalentes? Elas fazem um par?". Quando o participante respondia que sim, a experimentadora clicava na tela, fazendo abrir os pares de comparação e dava a instrução "Então escolha um outro par que é equivalente". Quando o participante respondia que os pares não eram equivalentes, a experimentadora clicava na tela, fazendo abrir os pares de comparação e dava a outra instrução "Então escolha um outro par que não é equivalente". Depois de fornecida a instrução verbal, o Teste de Analogia foi reapresentado.

Fidedignidade dos dados. Todos os dados foram coletados com o uso do programa MestreLibras (Elias 

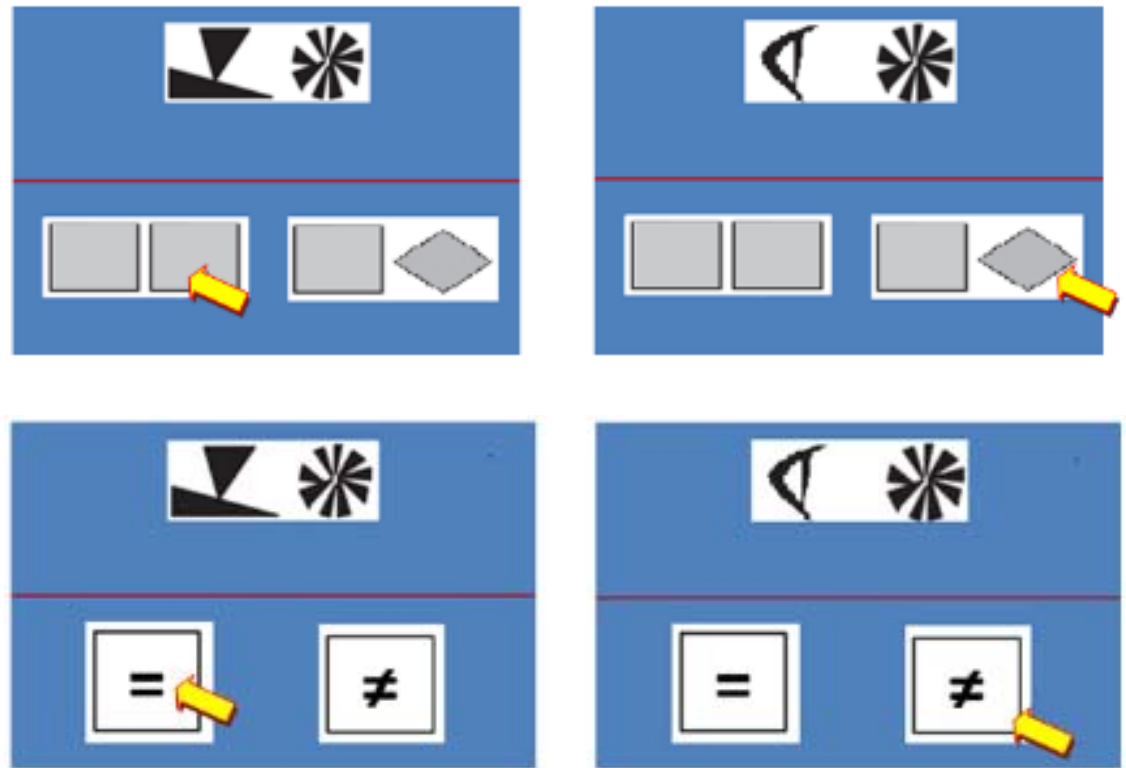

Figura 2. Nas duas figuras superiores, exemplos de tentativa de ensino com figuras geométricas. A esquerda, um exemplo de Eq-Eq (A1B1 - D1D1) À direita, um exemplo de NEq-NEq (A2B1 - D1D2). Nas duas figuras inferiores, exemplos de tentativa de ensino com os sinais de igual e diferente. À esquerda, um exemplo de Eq-Eq (A1B1 - Xi). À direita, um exemplo de NEq-NEq (A2B1 - Xd). As setas amarelas indicam o estímulo de comparação correto.

\& Goyos, 2010), que registrou todas as respostas automaticamente.

Integridade procedural. Aproximadamente $40 \%$ das sessões foram acompanhadas por um observador treinado, que registrou o comportamento da experimentadora durante as sessões de ensino e de teste. Os registros indicaram que a experimentadora seguiu corretamente o procedimento descrito em $100 \%$ das sessões observadas.
Aspectos éticos. O projeto foi encaminhado para o Comitê de Ética em Pesquisas em Seres Humanos da Universidade Federal de São Carlos. Após a aprovação (parecer número 997.119). Os participantes foram recrutados e receberam o Termo de Consentimento Livre e Esclarecido. Foram, então, agendados os dias de coleta com aqueles participantes que assinaram e concordaram em participar da pesquisa, de acordo com as suas disponibilidades.

\section{RESULTADOS}

Os resultados foram analisados de acordo com o delineamento, ou seja, para verificar quais os efeitos de cada condição experimental na emergência de respostas de analogia para relações de Eq-Eq e NEq-NEq. Foi feita também uma análise para comparar os desempenhos no Teste de Analogia dos participantes dos grupos 1 e 2 que passaram pelos procedimentos de facilitação.

A Tabela 4 apresenta o número de tentativas nas tarefas de ensino e de teste a que cada participante foi exposto e o número médio de tentativas para cada tipo de treino, sendo que o número mínimo de tentativas de ensino foi de 42 tentativas (nove $\mathrm{AB}$, nove $\mathrm{AC}, 12 \mathrm{AB} / \mathrm{AC}$ e 12 de facilitação) e o número mínimo para a formação das classes foi de 66 tentativas (nove $\mathrm{AB}$, nove $\mathrm{AC}, 12 \mathrm{AB} / \mathrm{AC}$, nove $\mathrm{BA}$, nove $\mathrm{CA}$, nove $\mathrm{BC}$ e nove $\mathrm{CB}$ ). $\mathrm{O}$ número médio total de tentativas entre os participantes foi de 169, variando de 57 a 324. Todos os participantes tiveram que repetir a execução de pelo menos uma tarefa. Em geral, o número de tentativas para alcance de critério foi menor para as relações AC (27 tentativas) do que para as relações AB (36 tentativas).
A Figura 3 apresenta a porcentagem de acertos dos participantes nos testes de analogia ao longo do procedimento; a linha pontilhada horizontal indica o critério mínimo de aprendizagem. O primeiro painel apresenta os resultados dos participantes que alcançaram o critério após o ensino $\mathrm{AB} / \mathrm{AC}$ ou após os testes das relações de equivalência. $\mathrm{O}$ segundo e o terceiro painéis apresentam o desempenho dos participantes que passaram pelos procedimentos de Facilitação 1 ou 2, respectivamente, e aqueles que responderam após a apresentação da instrução verbal.

Conforme pode ser observado no primeiro painel da Figura 3, P12 e P24 foram os únicos participantes que alcançaram o critério no teste de analogia imediatamente após o ensino das relações $\mathrm{AB} / \mathrm{AC}$. Dez participantes alcançaram o critério nesse teste após os testes das relações simétricas, transitivas e equivalentes. O segundo painel mostra que cinco (P3, P4, P14, P15, P17) de oito participantes $(62,5 \%)$ alcançaram o critério no Teste de Analogia após serem expostos ao procedimento de Facilitação 1. No terceiro painel, verifica-se que três (P9, P20, P22) de nove 
Número de tentativas de cada relação $A B, A C, B A, C A, B C$ e $C B$ e do procedimento de facilitação a que cada participante foi exposto por ordem crescente do número total de tentativas.

\begin{tabular}{|c|c|c|c|c|c|c|c|c|c|}
\hline \multirow{2}{*}{ Participante } & \multicolumn{7}{|c|}{ Relações } & \multirow{2}{*}{ Facilitação } & \multirow{2}{*}{ Tota } \\
\hline & $\mathbf{A B}$ & AC & ABAC & BA & CA & BC & CB & & \\
\hline $\mathrm{P} 12$ & 27 & 18 & 12 & --- & --- & --- & --- & --- & 57 \\
\hline P24 & 45 & 18 & 12 & --- & --- & --- & --- & --- & 75 \\
\hline $\mathrm{P} 2$ & 18 & 9 & 12 & 9 & 9 & 9 & 9 & --- & 75 \\
\hline P16 & 18 & 9 & 12 & 9 & 9 & 9 & 9 & --- & 75 \\
\hline P17 & 18 & 9 & 12 & 9 & 9 & 9 & 9 & 12 & 87 \\
\hline P28 & 27 & 18 & 12 & 9 & 9 & 9 & 9 & --- & 93 \\
\hline P3 & 18 & 18 & 12 & 9 & 9 & 9 & 9 & 12 & 96 \\
\hline P6 & 18 & 18 & 24 & 9 & 9 & 9 & 9 & 12 & 108 \\
\hline P11 & 18 & 27 & 12 & 18 & 9 & 9 & 9 & 12 & 114 \\
\hline P8 & 45 & 18 & 12 & 9 & 9 & 9 & 9 & 12 & 123 \\
\hline P27 & 27 & 27 & 24 & 18 & 18 & 18 & 18 & --- & 150 \\
\hline P4 & 18 & 18 & 36 & 18 & 18 & 18 & 18 & 12 & 156 \\
\hline P30 & 27 & 36 & 24 & 18 & 18 & 18 & 18 & --- & 159 \\
\hline P19 & 27 & 27 & 24 & 18 & 18 & 18 & 18 & 12 & 162 \\
\hline P29 & 45 & 9 & 12 & 9 & 9 & 9 & 9 & 60 & 162 \\
\hline P13 & 45 & 27 & 24 & 18 & 18 & 18 & 18 & --- & 168 \\
\hline P26 & 36 & 45 & 24 & 18 & 18 & 18 & 18 & --- & 177 \\
\hline P23 & 36 & 36 & 24 & 18 & 18 & 18 & 18 & 12 & 180 \\
\hline P25 & 36 & 36 & 24 & 18 & 18 & 18 & 18 & 12 & 180 \\
\hline P10 & 36 & 18 & 60 & 18 & 18 & 18 & 18 & 12 & 198 \\
\hline P1 & 27 & 27 & 48 & 18 & 18 & 18 & 18 & 36 & 210 \\
\hline P15 & 63 & 27 & 24 & 18 & 18 & 18 & 18 & 24 & 210 \\
\hline P14 & 27 & 36 & 36 & 18 & 18 & 27 & 18 & 36 & 216 \\
\hline P7 & 54 & 36 & 48 & 18 & 18 & 18 & 18 & 12 & 222 \\
\hline P18 & 63 & 27 & 24 & 18 & 18 & 18 & 18 & 48 & 234 \\
\hline P9 & 36 & 18 & 36 & 36 & 36 & 36 & 36 & 12 & 246 \\
\hline P20 & 45 & 54 & 36 & 27 & 27 & 27 & 27 & 12 & 255 \\
\hline P22 & 45 & 54 & 36 & 27 & 27 & 27 & 27 & 12 & 255 \\
\hline P21 & 45 & 54 & 36 & 27 & 27 & 27 & 27 & 36 & 279 \\
\hline P5 & 108 & 36 & 36 & 27 & 27 & 27 & 27 & 36 & 324 \\
\hline Média & 37 & 27 & 26 & 17 & 16 & 17 & 16 & 17 & 169 \\
\hline
\end{tabular}

participantes $(33,3 \%)$ alcançaram o critério no Teste de Analogia após a exposição ao procedimento de Facilitação 2.

Além disso, dos sete participantes que foram expostos à instrução, seis (P5, P6, P7, P8, P19 e P21) participantes $(85,7 \%)$ alcançaram o critério no Teste de Analogia aplicado imediatamente em seguida. Os participantes P1 e P10 não passaram pela fase da Instrução 1 , mesmo sem atingir o critério de desempenho do Teste de Analogia após o procedimento de facilitação, uma vez que solicitaram a saída do experimento.
A Tabela 5 apresenta as porcentagens de acertos por participante nos testes de analogia, separados por Eq-Eq e NEq-NEq. Observa-se que houve mais acertos nas relações Eq-Eq do que nas relações NEq-NEq no primeiro Teste de Analogia. Esse quadro se alterou de forma gradual ao longo do experimento, de modo que os participantes passaram a emitir mais respostas corretas nas relações NEq-NEq do que nas relações Eq-Eq antes de alcançarem o critério de desempenho no Teste de Analogia para os dois tipos de relações entre relações. 


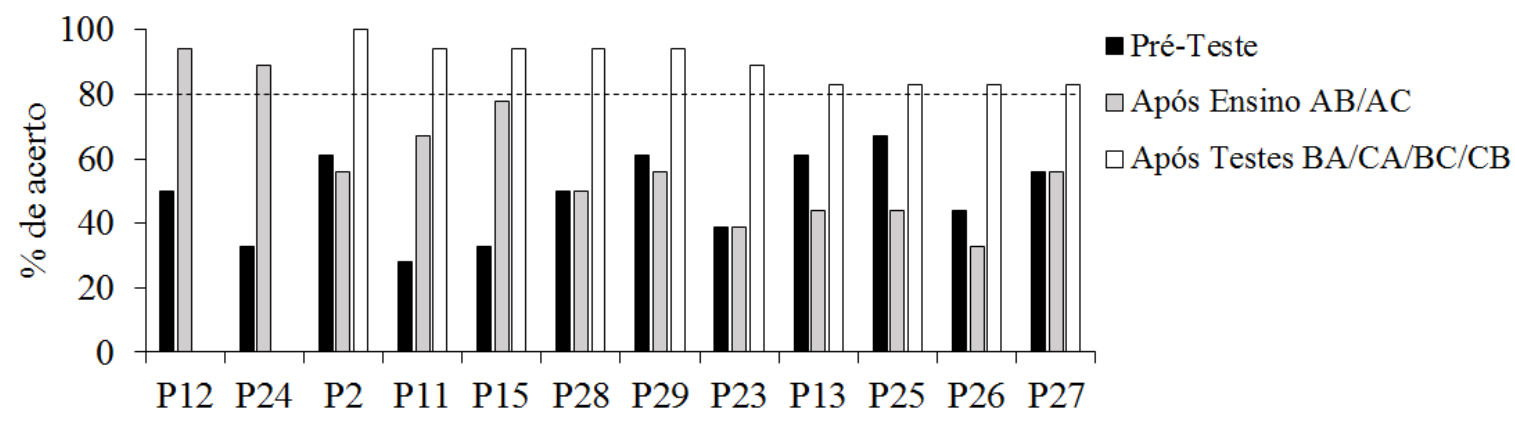

Participantes

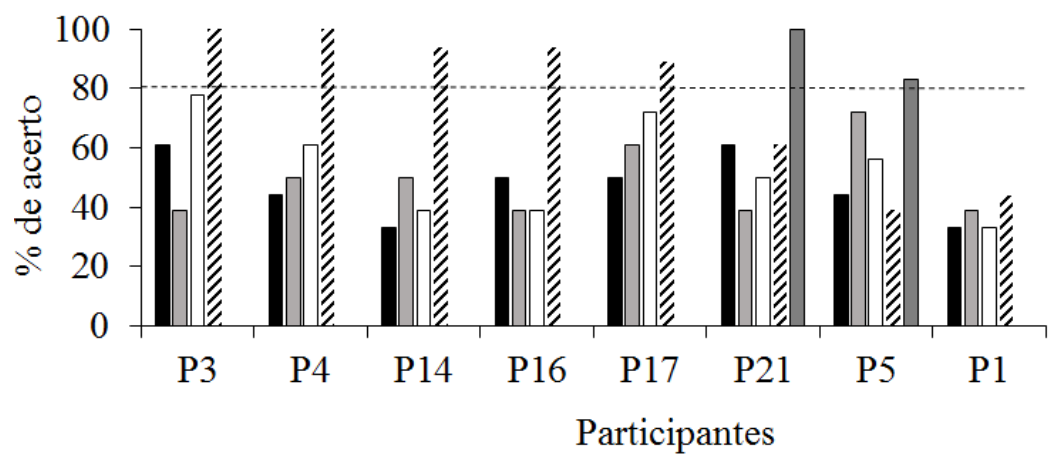

\author{
- Pré-Teste \\ $\square$ Após Ensino AB/AC \\ $\square$ Após Testes BA/CA/BC/CB \\ " Após Facilitação 1 \\ $\square$ Após Regra
}

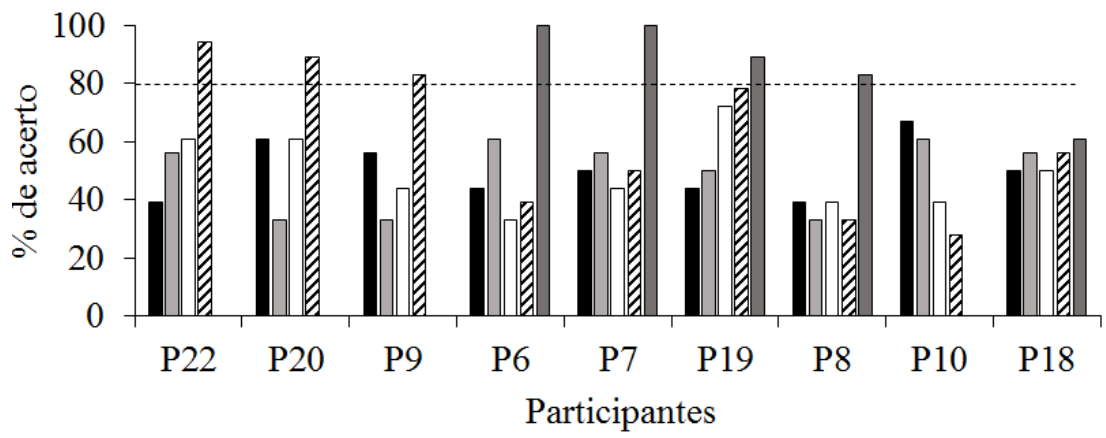

- Pré-Teste

$\square$ Após Ensino AB/AC

$\square$ Após Testes BA/CA/BC/CB

\Após Facilitação 2

$\square$ Após Regra

Figura 3. Porcentagem de acertos, para cada participante, nos testes de analogia ao longo do procedimento. As linhas pontilhadas indicam o critério de $80 \%$ de respostas corretas.

\title{
DISCUSSÃO
}

Os resultados indicam que dois participantes (P12 e P24) alcançaram o critério no Teste de Analogia após alcançarem o critério nos blocos de treino das relações de linha de base $\mathrm{AB}$ e $\mathrm{AC}$; e dez participantes (P2, P11, P13, P15, P23, P25, P26, P27, P28 e P29) após alcançarem o critério nos testes das relações emergentes. Esses dados replicam aqueles encontrados por Pérez et al. (2011) em que quatro de 12 participantes alcançaram critério no Teste de Analogia após alcançarem o critério no treino das relações $\mathrm{AB}$ e $\mathrm{AC}$ e no teste das relações $\mathrm{CB}$. A introdução dos testes de todas as relações emergentes de simetria e de transitividade não produziu diferença nos resultados do presente estudo em relação ao de Pérez et al., que testaram somente as relações CB. Portanto, nos dois estudos, aproximadamente entre $30 \%$ e $40 \%$ dos participantes alcançaram o critério no teste de analogia imediatamente após serem expostos ao treino ou ao treino e teste das relações condicionais. Esses dados indicam que, para a maioria dos participantes nos dois estudos, a formação das classes não é suficiente para a emergência das relações de analogia (Barnes et al.,1997; Stewart et al., 2001).

De acordo com os resultados nos testes de analogia, pode-se inferir que o procedimento de Facilitação $1 \mathrm{com}$ figuras geométricas foi mais eficiente em promover as relações de analogia do que o procedimento de Facilitação 2 com os sinais igual/diferente. Isso pode se dever ao fato de que a tarefa do procedimento de Facilitação 1 apresenta configurações de estímulos semelhantes ao Teste de Analogia, em que os estímulos de comparação foram pares de figuras, e não símbolos isolados. No estudo de Pérez et al. (2011), 40\% dos participantes que passaram pelo procedimento de facilitação com figuras geométricas 
Porcentagens de acertos por participante nos testes de analogia separados por Eq-Eq e NEq-NEq. As células em cinza indicam que houve mais acertos nas relações $E q-E q$

\begin{tabular}{|c|c|c|c|c|c|}
\hline \multirow{3}{*}{ Participante } & \multicolumn{5}{|c|}{ Teste de Analogia } \\
\hline & 1 & 2 & 3 & 4 & 5 \\
\hline & \multicolumn{5}{|c|}{$\%$ acertos Eq-Eq / \% acertos NEq-NEq } \\
\hline $\mathrm{P} 1$ & $0 / 67$ & $44 / 33$ & $22 / 44$ & $33 / 56$ & --- \\
\hline $\mathrm{P} 2$ & $67 / 56$ & $44 / 67$ & $100 / 100$ & --- & --- \\
\hline P3 & $56 / 67$ & $33 / 44$ & $89 / 67$ & $100 / 100$ & --- \\
\hline P4 & $56 / 33$ & $67 / 33$ & $67 / 56$ & $100 / 100$ & --- \\
\hline P5 & $44 / 44$ & $67 / 78$ & $44 / 67$ & $11 / 67$ & $67 / 100$ \\
\hline P6 & $56 / 33$ & $56 / 67$ & $11 / 56$ & $22 / 56$ & $100 / 100$ \\
\hline $\mathrm{P} 7$ & $56 / 44$ & $44 / 67$ & $33 / 56$ & $44 / 56$ & $100 / 100$ \\
\hline P8 & $44 / 33$ & $11 / 56$ & $22 / 56$ & $11 / 56$ & $78 / 89$ \\
\hline P9 & $56 / 56$ & $22 / 44$ & $67 / 22$ & $78 / 89$ & $100 / 100$ \\
\hline P10 & $78 / 56$ & $67 / 56$ & $22 / 56$ & $22 / 33$ & --- \\
\hline P11 & $44 / 11$ & $56 / 78$ & $89 / 100$ & $100 / 100$ & --- \\
\hline $\mathrm{P} 12$ & $44 / 56$ & $89 / 100$ & --- & --- & --- \\
\hline P13 & $78 / 44$ & $44 / 44$ & $78 / 89$ & --- & --- \\
\hline P14 & $33 / 33$ & $33 / 67$ & $33 / 44$ & $89 / 100$ & --- \\
\hline P15 & $33 / 67$ & $33 / 44$ & $22 / 56$ & $89 / 100$ & --- \\
\hline P16 & $56 / 11$ & $100 / 56$ & $100 / 89$ & --- & --- \\
\hline P17 & $33 / 67$ & $44 / 78$ & $78 / 67$ & $89 / 89$ & --- \\
\hline P18 & $56 / 44$ & $56 / 56$ & $33 / 67$ & $56 / 56$ & $78 / 44$ \\
\hline P19 & $56 / 33$ & $44 / 56$ & $78 / 67$ & $67 / 89$ & $89 / 89$ \\
\hline P20 & $67 / 56$ & $22 / 44$ & $56 / 67$ & $78 / 100$ & --- \\
\hline P21 & $44 / 78$ & $44 / 33$ & $22 / 78$ & $44 / 78$ & $100 / 100$ \\
\hline P22 & $11 / 67$ & $33 / 78$ & $56 / 67$ & $89 / 100$ & --- \\
\hline P23 & $44 / 33$ & $44 / 33$ & $89 / 89$ & $100 / 100$ & --- \\
\hline P24 & $33 / 33$ & $89 / 89$ & $89 / 100$ & --- & --- \\
\hline P25 & $89 / 44$ & $44 / 44$ & $78 / 89$ & $100 / 100$ & --- \\
\hline P26 & $67 / 22$ & $33 / 33$ & $78 / 89$ & --- & --- \\
\hline P27 & $67 / 44$ & $44 / 67$ & $100 / 67$ & --- & --- \\
\hline P28 & $67 / 33$ & $44 / 56$ & $100 / 89$ & --- & --- \\
\hline P29 & $67 / 67$ & $33 / 67$ & $33 / 44$ & $33 / 67$ & $89 / 100$ \\
\hline P30 & $67 / 56$ & $56 / 56$ & $100 / 89$ & --- & --- \\
\hline
\end{tabular}

melhoraram o desempenho no Teste de Analogia; no presente estudo, $62,5 \%$ dos participantes expostos ao procedimento de Facilitação 1 melhoraram o desempenho no Teste de Analogia. Essa diferença pode ser função da história experimental construída nesse estudo, em que os participantes foram expostos a testes adicionais das relações emergentes, incluindo relações de simetria e de transitividade.

O maior número de acertos para as relações NEq-NEq do que nas relações Eq-Eq (ver Tabela 5) pode indicar que o controle para responder às relações Eq-Eq foi mais complexo (o participante deveria ficar sob controle de todos os termos da analogia) e, portanto, foi fortalecido gradualmente em função do aprendizado derivado das diferentes tarefas.
Essa melhora gradual no desempenho dos participantes tem sido observada extensamente nos experimentos que usam o procedimento de MTS para o ensino das relações condicionais de linha de base e para o teste de relações emergentes na formação de classes de estímulos equivalentes (e.g., McIlvane \& Dube, 2003; Mcllvane, Serna, Dube, \& Stromer, 2000). Uma hipótese que pode explicar em parte os achados deste estudo em particular, principalmente no que diz respeito ao maior número observado de respostas corretas em relações NEq-NEq do que nas relações EqEq, pode estar relacionada ao controle exercido pelos estímulos sobre a resposta de escolha em tentativas de MTS (Wilkinson \& McIlvane, 1997). Por exemplo, o participante pode emitir a resposta de escolha do estímulo 
de comparação $\mathrm{B} 1$ na presença do modelo $\mathrm{A} 1$ porque a relação aprendida é: "Quando A1, então eu rejeito B2", e não a relação esperada "Quando A1, então eu seleciono B1". Nesse caso, de controle por rejeição de comparação incorreta, a relação formada entre os estímulos é a relação A1B2, e não a relação esperada A1B1, mesmo que a resposta de escolha observada seja exatamente a mesma em ambos os casos. Assim, se os participantes desse experimento estiverem rejeitando o estímulo de comparação incorreto, as relações formadas podem ser, em sua maioria, NEqNEq. O controle por rejeição poderia explicar os dados encontrados, principalmente porque o experimento utilizou tentativas com apenas dois estímulos de comparação, o que, segundo de Rose, Hidalgo e Vasconcellos (2013), torna mais provável que respostas por rejeição do estímulo de comparação incorreto sejam registradas como corretas. Para controlar essa variável, estudos futuros podem introduzir um terceiro estímulo de comparação (Perez \& Millan, 2014), diminuindo a chance de controle por rejeição e aumentando a probabilidade de formação de classes equivalentes, como usado no experimento de Arantes e de Rose (2015).

Além disso, esses resultados também podem indicar controle de características irrelevantes da contingência. Por exemplo, o desempenho dos participantes poderia estar sob controle da relação entre um estímulo de cada par, como, por exemplo, relacionar A1B1 (par equivalente) com B2C1 (par não equivalente) e não com A2B2 (par equivalente), em função de $\mathrm{A} 1$ já ter sido relacionado anteriormente com C1, nas fases de ensino. No estudo de Goswami e Brown (1990), os autores notaram que os participantes respondiam ao problema "Aranha:Teia::Abelha: Colmeia/ Mel/Formiga/Mosca" ("aranha está para teia assim como abelha está para?"), selecionando colmeia (em detrimento de mel, formiga ou mosca) pela relação entre teia e colmeia, sendo que os termos "a" (aranha) e "c" (abelha) não tiveram influência no responder.

De maneira geral, os resultados desse estudo indicam que somente o ensino das relações condicionais de linha de base $\mathrm{AB}$ e $\mathrm{AC}$ parece não ser suficiente para gerar o repertório emergente de analogia e que a apresentação da instrução verbal foi bastante eficiente nesse sentido. Esses dados diferem do Experimento 2 de Barnes et al. (1997), no qual os participantes passaram no teste de analogia sem precisarem responder aos testes das relações emergentes. Entretanto, é preciso lembrar que há diferenças entre os dois estudos e que nenhum participante do Experimento 2 de Barnes et al. alcançou o critério no Teste de Analogia na primeira exposição. No estudo desses autores, os participantes eram expostos repetidamente aos blocos de ensino e aos testes de analogia até alcançarem critério nos testes. Outra diferença é que esses autores utilizaram tentativas de ensino com MTS com três estímulos de comparação.

Retomando a definição original de analogia, proposta por Barnes et al. (1997), como relacionar relações de equivalência a outras relações de equivalência e relações de não-equivalência a outras relações de nãoequivalência, pode-se inferir que os participantes deste estudo apresentaram, em momentos diferentes, respostas de analogia sem que fossem diretamente ensinadas. Esse dado corrobora a proposta de outros estudos sobre analogia (e.g., Stewart \& Barnes-Holmes, 2001; Stewart et al., 2001) de que seres humanos verbais, após exposição prolongada a certas contingências de reforçamento fornecidas pela comunidade verbal, demonstram responder com base nas relações derivadas, definidas por dicas contextuais adicionais. Nesse estudo, pode-se interpretar que as dicas contextuais de "pertencer à mesma classe" ou "pertencer a classes diferentes" exerceram controle sobre o responder nos testes de analogia.

Finalmente, os dados obtidos nesse estudo estão de acordo com o que se encontra na literatura sobre raciocínio analógico e equivalência de estímulos. A exceção é o fato de que 20 dos 29 participantes falharam nos testes de relações de equivalência mesmo após bom desempenho no ensino das relações condicionais $\mathrm{AB}$ e AC (ver Tabela 4). Uma hipótese é que o Teste de Analogia estava funcionando como uma tarefa distratora entre as fases de ensino e de teste das relações de equivalência. Arntzen e Vie (2013) demonstraram que a introdução de tarefas distratoras diminui a acurácia do desempenho em procedimentos de MTS em estudos sobre equivalência de estímulos. Para estudos futuros, seria interessante avaliar essa questão para evitar vieses no procedimento, como, por exemplo, aplicar os testes de relações e equivalência antes de introduzir o Teste de Analogia. Estudos futuros poderiam incluir, por exemplo, uma tarefa inicial de analogia, em que os pares de estímulos seriam compostos por figuras familiares (uma maçã e uma laranja / um cachorro e um gato) e as respostas corretas fossem reforçadas, para garantir que os participantes entendam a tarefa. Além disso, os participantes poderiam ser expostos aos dois procedimentos de facilitação antes de introduzir a instrução verbal no último Teste de Analogia. Outro desafio na área é a transposição desse modelo experimental para a sua utilização em procedimentos de pesquisa sobre raciocínio analógico e analogia em ambientes mais naturais e para o ensino de repertórios socialmente relevantes.

\section{REFERÊNCIAS}

Arantes, A., \& de Rose, J. C. (2015). High probability of equivalence class formation with both Sample-S+ and Sample-Scontrolling relations in baseline. The Psychological Record, 65(4), 743-748. https://doi.org/10.1007/s40732-015-0143-2
Arntzen, E., \& Vie, A. (2013). The expression of equivalence classes influenced by distractors during DMTS test trials. European Journal of Behavior Analysis, 14(1), 151-164. https://doi.org $/ 10.1080 / 15021149.2013 .11434453$ 
Barnes, D., Hegarty, N., \& Smeets, P. M. (1997). Relating equivalence relations to equivalence relations: A relational framing model of complex human functioning. The Analysis of Verbal Behavior, 14(1), 57-83. https://doi.org/10.1007/ BF03392916

Carpentier, F., Smeets, P. M., Barnes-Holmes, D., \& Stewart, I. (2004). Matching derived functionally-same relations: Equivalence-equivalence and classical analogies. The Psychological Record, 54(2), 255-273. https://doi.org/10.1007/ BF03395473

de Rose, J. C., Hidalgo, M., \& Vasconcellos, M. (2013). Controlling relations in baseline conditional discriminations as determinants of stimulus equivalence. The Psychological Record, 63(1), 85-98. doi:10.11133/j.tpr.2013.63.1.007.

Elias, N. C., \& Goyos, C. (2010). MestreLibras no ensino de sinais: Tarefas informatizadas de escolha de acordo com o modelo e equivalência de estímulos. Em E. G. Mendes \& M. A. Almeida (Orgs.), Das margens ao centro: Perspectivas para as políticas e práticas educacionais no contexto da educação especial inclusiva (pp. 223-234). Araraquara: Junqueira \& Marin Editora,

Goswami, U., \& Brown, A. L. (1990). Higher-order structure and relational reasoning: Contrasting analogical and thematic relations. Cognition, 36(3), 207-226. https://doi. org/10.1016/0010-0277(90)90057-Q

Kolodner, J. L. (1997). Educational implications of analogy: A view from case-based reasoning. American Psychologist, 52(1), 57. http://dx.doi.org/10.1037/0003-066X.52.1.57

Lipkens, R., \& Hayes, S. C. (2009). Producing and recognizing analogical relations. Journal of the Experimental Analysis of Behavior, 91(1), 105-126. doi: 10.1901/jeab.2009.91-105

McIlvane, W. J., \& Dube, W. V. (2003). Stimulus control topography coherence theory: Foundations and extensions. The Behavior Analyst, 26(2), 195-213. https://doi.org/10.1007/BF03392076

McIlvane, W. J., Serna, R., Dube,W. V., \& Stromer, R. (2000). Stimulus control topography coherence and stimulus equivalence: Reconciling test outcomes with theory. Em J. Leslie \& D. E. Blackman (Eds.), Issues in experimental and applied analysis of human behavior (pp. 85-110). Reno, NV: Context Press.
Pérez, V., García, A., \& Gómez, J. (2011). Facilitation of the equivalence-equivalence response. Psicothema, 23(3), 407414.

Perez, W. F., \& Millán, L. V. R. (2014). Indução de controles por seleção e por rejeição em tarefas de emparelhamento com o modelo: Uma revisão metodológica. Acta Comportamentalia, 22(2), 227-242.

Sidman, M. (1971). Reading and auditory-visual equivalences. Journal of Speech and Hearing Research, 14(1), 5-13. http:/ dx.doi.org/10.1044/jshr.1401.05

Sidman, M., \& Tailby, W. (1982). Conditional discrimination vs. matching to sample: An expansion of the testing paradigm. Journal of the Experimental Analysis of Behavior, 37(1), 5-22. https://doi.org/10.1901/jeab.1982.37-5

Skinner, B. F. (1957). Verbal behavior. New York: AppletonCentury-Crofts.

Stewart, I., \& Barnes-Holmes, D. (2001). Understanding metaphor: A relational frame perspective. The Behavior Analyst, 24(2), 191-199. https://doi.org/10.1007/BF03392030

Stewart, I., Barnes-Holmes, D., Hayes, S. C., \& Lipkens, R. (2001). Relations among relations: Analogy, metaphor, and allegory. Em S. C. Hayes, D. Barnes-Holmes \& B. Roche (Eds.), Relational frame theory: A post-Skinnerian account of human language and cognition (pp. 73-86). New York: Plenum.

Stewart, I., Barnes-Holmes, D., \& Roche, B. (2004). A functionalanalytic model of analogy using the relational evaluation procedure. The Psychological Record, 54(4), 531-552. https:// doi.org/10.1007/BF03395491

Stewart, I., Barnes-Holmes, D., Roche, B., \& Smeets, P. M. (2001). Generating derived relational networks via the abstraction of common physical properties: A possible model of analogical reasoning. The Psychological Record, 51(3), 381-408. https:// doi.org/10.1007/BF03395405

Stokes, T. F., \& Baer, D. M. (1977). An implicit technology of generalization. Journal of Applied Behavior Analysis, 10(2), 349-367. doi: 10.1901/jaba.1977.10-349

Wilkinson, K. M., \& McIlvane, W. J. (1997). Blank comparison analysis of emergent symbolic mapping by young children. Journal of Experimental Child Psychology, 67(2), 115-130. https://doi.org/10.1006/jecp.1997.2402 